\title{
Fibrosis subepitelial de grado IV como complicación de un entrecruzamiento acelerado pulsado de fibras de colágeno corneal transepitelial en un paciente pediátrico: reporte de caso y revisión de los factores asociados
}

\section{Severe haze as a complication of pulsed-light accelerated transepithelial corneal cross-linking in a pediatric patient: a case report and review of associated factors}

\author{
Oscar Fernández-Vizcaya*, Alejandro Babayán-Sosa, Regina Velasco-Ramos, Julia C. Pacheco-del-Valle, \\ Elisa Alegría-Gómez, Arturo Abascal-Espino y Oscar Baca-Lozada \\ Departamento de Córnea y Cirugía Refractiva, Fundación Hospital Nuestra Señora de la Luz, Ciudad de México, México
}

\begin{abstract}
Resumen
El entrecruzamiento de fibras de colágeno corneales o crosslinking, una técnica que ha cobrado cada vez más popularidad ya que ha demostrado desacelerar la progresión del queratocono, ha evolucionado en relación con el tiempo de aplicación, la posibilidad de mantener el epitelio, la cantidad de energía y las edades a las que se aplica, observándose en la mayoría de los casos buenos resultados con pocas complicaciones. Presentamos el caso de un paciente en edad pediátrica que desarrolló una fibrosis subepitelial de grado IV posterior a un procedimiento de crosslinking transepitelial pulsado acelerado. Se hace una revisión de los posibles factores desencadenantes de esta fibrosis antes y después de aplicar el tratamiento.
\end{abstract}

Palabras clave: Transepitelial. Colágena. Córnea. Crosslinking. Fibrosis subepitelial. Riboflavina.

\begin{abstract}
Corneal crosslinking, a technique that has become increasingly popular since it slows the progression of keratoconus, has evolved regarding the time of application, possibility of maintaining the epithelium, amount of energy and ages in which is applied, observing in the majority of cases good results with few complications. We present the case of a pediatric patient in which a severe haze developed after an accelerated pulsed transepithelial corneal crosslinking. We present a review of the possible contributing factors so they can be considered before and after applying this treatment.
\end{abstract}

Key words: Transepithelial. Collagen. Cornea. Crosslinking. Haze. Riboflavin.

Correspondencia:

*Oscar Fernández-Vizcaya

Ezequiel Montes 135

Col. Tabacalera

Fecha de recepción: 17-06-2020

C.P. 06030, Ciudad de México, México

E-mail: droscarfdez@gmail.com

0187-4519/( 2020 Sociedad Mexicana de Oftalmología. Publicado por Permanyer. Este es un artículo open access bajo la licencia CC BY-NC-ND (http://creativecommons.org/licenses/by-nc-nd/4.0/).
Disponible en internet: 13-04-2021 Rev Mex Oftalmol. (ahead of print) www.rmo.com.mx
Fecha de aceptación: 29-10-2020 


\section{Introducción}

El entrecruzamiento de fibras de colágeno corneales o crosslinking, una técnica desarrollada en la universidad de Dresden y aplicada clínicamente por primera vez en 2003 por Wollensak, Spoerl y Seiler, quienes lograron una estabilización de la progresión del queratocono en el $70 \%$ de los casos a 5 años ${ }^{1,2}$, es hasta el momento el único tratamiento capaz de retrasar la progresión del queratocono ${ }^{3}$. Existen numerosas publicaciones sobre su eficacia combinada con otros procedimientos ${ }^{4-8}$ y sus pocas complicaciones, explicadas en su gran mayoría por variantes específicas en el tratamiento, así como por características del queratocono y de cicatrización del paciente ${ }^{9}$.

Dentro de la evolución del crosslinking, se ha reducido el tiempo de aplicación del tratamiento aumentando la energía ${ }^{10}$ y se han explorado otras variables, como la posibilidad de mantener el epitelio durante el mismo ${ }^{11}$. La eficacia de este último ha sido cuestionada con reportes a favor y en contra ${ }^{12}$, tratando a algunos pacientes con parámetros de grosor corneal menores que los descritos en el protocolo original de Dresden. Además de la penetración hasta capas profundas, se deben tener en cuenta las complicaciones relacionadas con la superficie ocular, por lo que presentamos un caso en el que se desarrolló una fibrosis subepitelial de grado IV posterior a un procedimiento crosslinking transepitelial pulsado acelerado.

\section{Caso clínico}

Paciente de sexo masculino, de 15 años de edad, que refiere una disminución progresiva de la agudeza visual de 1 año de evolución, con antecedentes sistémicos de asma bronquial en tratamiento con fluticasona de 7 años de evolución, actualmente controlada. Como antecedentes oftalmológicos refiere un cuadro de conjuntivitis alérgica no especificada manejada 2 años atrás con olopatadina al $0.2 \%$ cada 24 horas y hialuronato de sodio al $0.4 \%$ sin conservadores durante 1 mes, posterior a lo cual no tuvo recurrencias ni recibió otro tratamiento.

En la exploración oftalmológica presenta una agudeza visual sin corrección (AVSC) en el ojo derecho (OD) de 20/200 y en el ojo izquierdo (OI) de 20/400, con refracciones OD $-2.00=-5.00 \times 170^{\circ}$ y Ol $-3.00=$ $-7.00 \times 15^{\circ}$, y una agudeza visual con corrección (AVCC) en el OD de 20/50 y en el Ol de 20/80. Párpados y superficie ocular sin alteraciones (se buscaron intencionadamente datos relacionados con los antecedentes alérgicos). Segmentos anterior y posterior, presión ocular y fondo de ojo sin alteraciones. Se documenta, gracias a una refracción previa que proporciona el paciente, un aumento del equivalente esférico y queratometría máxima de más de 1.5 dioptrías en ambos ojos en 6 meses, que aunado a la disminución de la agudeza visual referida en el último año y el grosor residual limítrofe apoyaron la decisión para un crosslinking a pesar de no contar con un seguimiento topográfico por la posibilidad de no contar con un grosor suficiente en visitas subsecuentes (Fig. 1).

Se le realizó un entrecruzamiento acelerado pulsado de fibras de colágeno corneal en el OI, sin complicaciones ni pérdida de líneas de agudeza visual; sin embargo, debido al dolor y a molestias posteriores al tratamiento sin epitelio, el paciente decidió considerar la opción del entrecruzamiento transepitelial para el OD, explicando previamente a él y sus familiares que, además de los riesgos del procedimiento, aún existe controversia en relación con los resultados a largo plazo del entrecruzamiento transepitelial comparado con el convencional. El tratamiento en el OD se realizó 4 meses después del primer entrecruzamiento, bajo anestesia tópica, colocando ParaCel ${ }^{\mathrm{TM}}$ (riboflavina al $0.25 \%$ con cloruro de benzalconio, EDTA, trometamol e hidroxipropilmetilcelulosa) para atravesar el epitelio cada 2 minutos durante 8 minutos. Se irrigó la superficie para retirar el ParaCel ${ }^{\mathrm{TM}}$ y la córnea se expuso a luz UVA (Sistema KXL, Avedro Inc., Waltham, MS, USA) mientras se colocó VibeX Xtra ${ }^{\mathrm{TM}}$ (riboflavina al $0.22 \%$ ) durante 5 minutos y 20 segundos a una irradiancia de $45 \mathrm{~mW} / \mathrm{cm}^{2}$ ( $1 \mathrm{~s}$ on y $1 \mathrm{~s}$ off) para una dosis total de área de $7.2 \mathrm{~J} / \mathrm{cm}^{2}$. Al finalizar el tratamiento, el cirujano se percató de la presencia de un pequeño defecto epitelial puntiforme central y colocó un lente de contacto terapéutico. Se prescribió como tratamiento la colocación de gotas de carboximetilcelulosa sódica al 0.5\% cuatro veces al día, diclofenaco tópico al $0.1 \%$ cada 8 horas por 48 horas y gatifloxacino al $0.3 \%$ combinado con prednisolona al 1\% cuatro veces al día por 14 días.

En la revisión a los 7 días, el paciente refirió una baja visual importante en el OD, con una AVSC de contar dedos a $50 \mathrm{~cm}$, hiperemia conjuntival, lente de contacto terapéutico con múltiples depósitos de lípidos y proteínas, pérdida de la transparencia corneal, edema central y un defecto epitelial de $4 \mathrm{~mm}$ vertical por $5 \mathrm{~mm}$ horizontal (Fig. 2).

Se decidió retirar el lente de contacto, colocar un parche oclusivo (solo levantarlo para colocar medicamentos) y cambiar la carboximetilcelulosa por hialuronato de sodio sin conservadores al $0.4 \%$ 


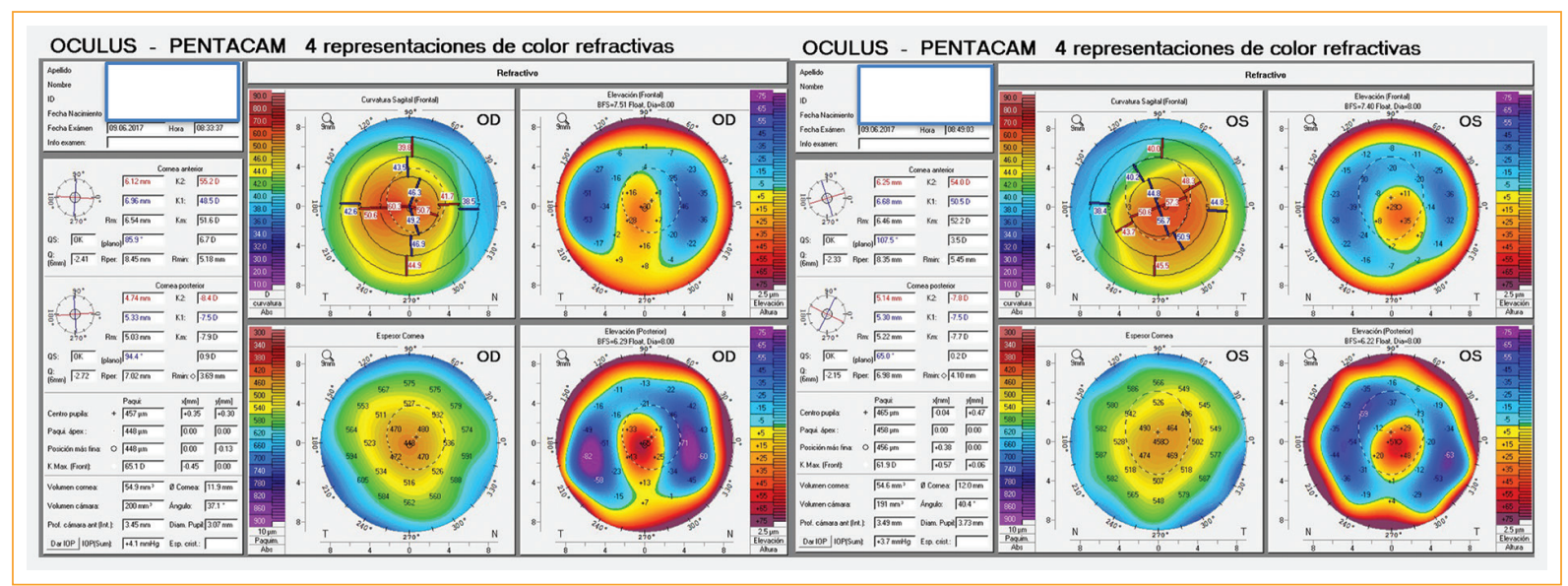

Figura 1. PENTACAM inicial que evidencia grosores en el límite para un tratamiento de crosslinking en ambos ojos.

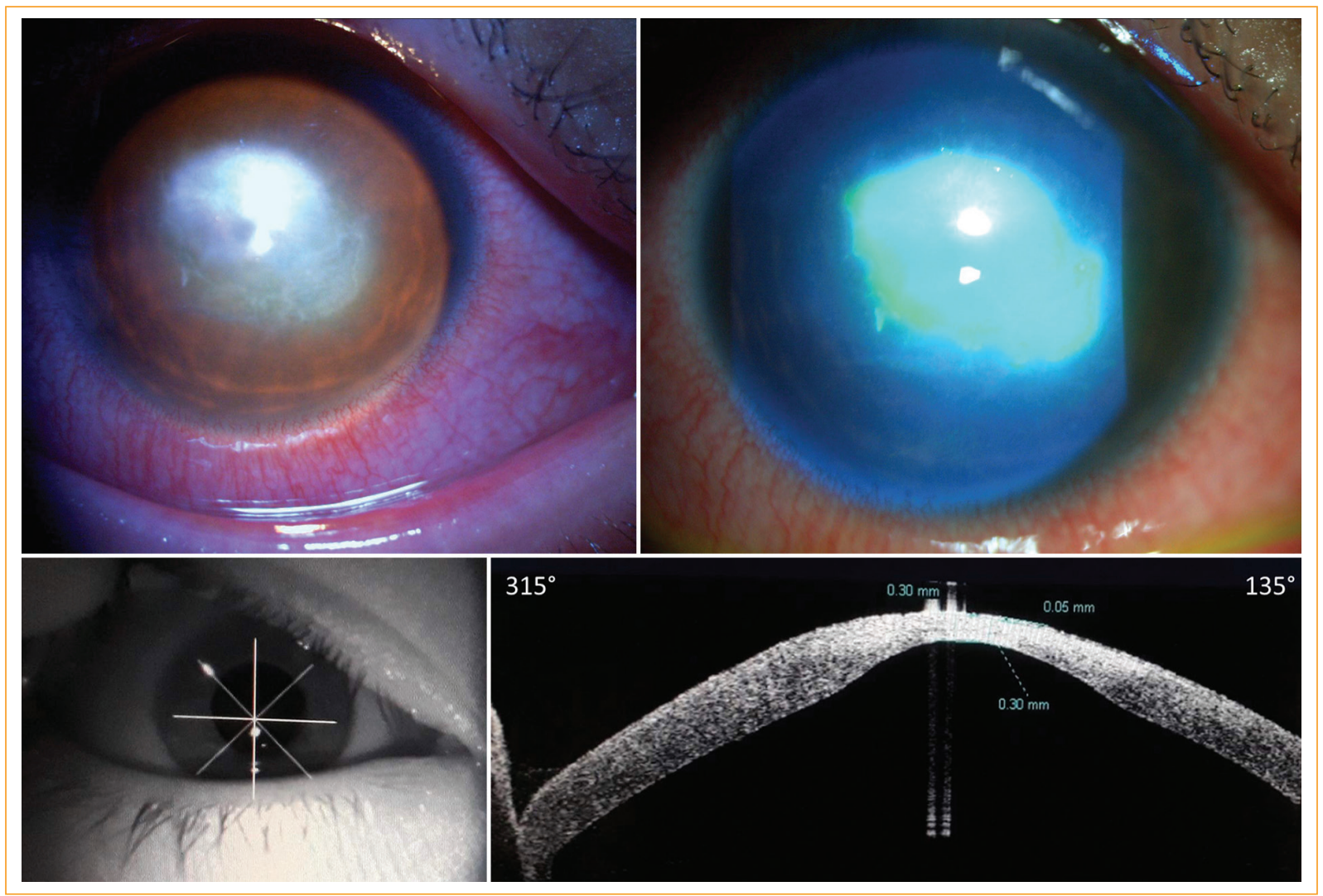

Figura 2. Defecto epitelial teñido con fluoresceína y tomografía de coherencia óptica de dominio tiempo en la que se observó un adelgazamiento corneal central con cambio importante en la curvatura posterior y una medida mínima central de $0.30 \mathrm{~mm}$.

cada 6 horas, trehalosa al 3\% cada 8 horas, y cambiar el antibiótico a netilmicina al $0.3 \%$ con dexametasona al $0.1 \%$ cada 6 horas, con seguimiento cada 24 horas (Fig. 3).
A las 72 horas se documentó el cierre completo del defecto. Un mes después presentó una AVSC de 20/400 y una paquimetría central por tomografía de coherencia óptica de $0.21 \mathrm{~mm}$. Se manejó con acetato 


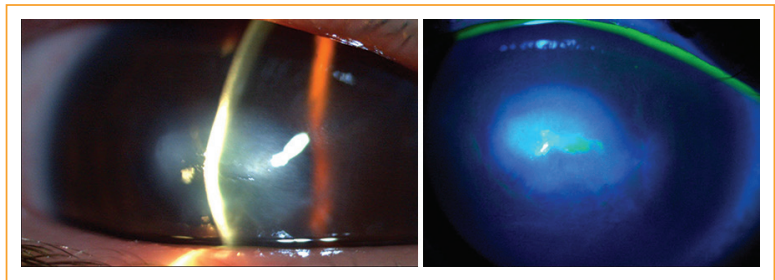

Figura 3. A las 48 horas del cambio de tratamiento se observa una reducción importante del defecto epitelial.

de prednisolona al $1 \%$ en una dosis reductiva los siguientes meses. A los 6 meses se mantenía la opacidad central a expensas de fibrosis subepitelial de grado IV, con una AVSC de 20/200, a pesar del tratamiento prolongado con esteroides. Se ofreció la posibilidad de un tratamiento de queratoplastia lamelar anterior profunda mediante láser de femtosegundos, el cual continúa pendiente, ya que el paciente no volvió a presentarse al departamento para el seguimiento de su evolución (Fig. 4).

\section{Discusión}

Las principales complicaciones documentadas en el entrecruzamiento con remoción del epitelio incluyen dolor ocular posoperatorio, fibrosis subepitelial, infiltrados estériles y queratitis infecciosas ${ }^{13}$. Se han realizado modificaciones para mejorar las molestias del paciente, al mantener el epitelio intacto utilizando sustancias o técnicas para que la riboflavina pueda difundirse al estroma, reduciendo los tiempos del tratamiento con una mayor intensidad de radiación (entrecruzamiento acelerado), con un menor porcentaje de edema estromal, infiltrados estériles, fibrosis subepitelial, queratitis infecciosas y pérdida de líneas de agudeza visual $^{14}$.

Aunque la seguridad parece ser mucho mejor manteniendo el epitelio, existe aún controversia sobre la eficacia de este tratamiento en las diferentes edades, observándose en microscopías confocales solo apoptosis superficial del estroma anterior ${ }^{15}$.

\section{Factores asociados a complicaciones y posible relación con el caso}

\section{1) Factores comunes a todos los tratamientos de entrecruzamiento}

Algunos factores a tener en cuenta en los tratamientos con remoción epitelial son valores

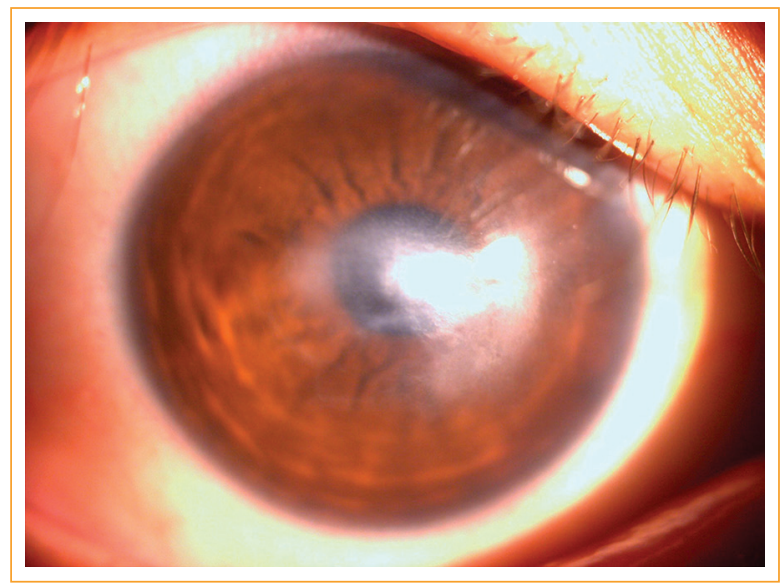

Figura 4. Fibrosis subepitelial grado IV, 6 meses después del tratamiento a pesar del tratamiento con esteroide tópico.

queratométricos elevados, paquimetrías delgadas, uso prolongado de antiinflamatorios no esteroideos (AINE) posquirúrgicos, blefaritis preoperatoria y conjuntivitis alérgica activa ${ }^{16}$. Estos factores pudieran ser considerados para nuestro caso a pesar de ser transepitelial; sin embargo, la conjuntivitis alérgica no se encontraba activa ni bajo tratamiento, siendo un factor siempre a descartar por la posibilidad de reactivación. La paquimetría se encontraba dentro del rango aceptado comúnmente para el tratamiento. Los AINE, que a pesar de su importancia y utilidad pueden retrasar la epitelización, causar lesiones, ulceración y hasta lisis con perforación ${ }^{17}$, solo se utilizaron durante 2 días posteriores al procedimiento; sin embargo, no se supervisaron en la etapa de su retiro el grado de epitelización ni el estado del lente de contacto hasta el día 7.

Entre los factores no siempre mencionados, pero que deben tomarse en cuenta previo a cualquier tratamiento en que se altere la superficie ocular, se encuentran el estado del limbo, las enfermedades inflamatorias, el ojo seco, la queratitis neurotrófica (antecedente de queratitis herpéticas), las alteraciones en el cierre y la frecuencia del parpadeo.

\section{2) Tipo de tratamiento transepitelial}

Otros factores de riesgo importantes son el tipo específico de tratamiento transepitelial y los hallazgos transoperatorios, incluyendo la presencia de defectos epiteliales puntiformes, ya sea por deshidratación, trauma directo o una mayor exposición a riboflavina con cloruro de benzalconio. Las 
variantes del tratamiento transepitelial más importantes a considerar para defectos epiteliales en el posoperatorio son el tipo de riboflavina, el tiempo en presencia de agentes epiteliotóxicos (como el cloruro de benzalconio), y la energía y el tiempo de la luz ultravioleta.

Taneri, et al. ${ }^{18}$ reportaron una mayor tasa de defectos epiteliales persistentes en tratamientos transepiteliales en el entrecruzamiento llamado Medio-Cross y en el aplicado únicamente con ParaCel ${ }^{\mathrm{TM}}$; ambos contienen riboflavina con cloruro de benzalconio y se realizan de manera continua, llegando a presentar defectos persistentes un $50-63 \%$ de los pacientes.

Debido a estas complicaciones, se establecieron tratamientos en dos pasos: ParaCel ${ }^{\mathrm{TM}}$ en un primer tiempo y VibeX Xtra $^{\text {TM }}$ (riboflavina sin cloruro de benzalconio y sin dextrano) en un segundo tiempo, ya sea con luz continua o pulsada, reportándose solo para el grupo de luz pulsada hasta un $5 \%$ de defectos epiteliales persistentes ${ }^{18}$. A este segundo grupo perteneció nuestro paciente, en el que revisamos y descartamos también la posibilidad de que el cirujano no hubiese cambiado el tipo de riboflavina para el segundo tiempo. Se ha especulado, además, que un mayor tiempo de desecación en el protocolo pulsado da como resultado un mayor trauma epitelial.

\section{3) Oxígeno}

La distribución del oxígeno a través de toda la córnea no es homogénea; la permeabilidad y el consumo de oxígeno varían entre las diferentes capas. Los radicales libres en el entrecruzamiento corneal creados por la irradiación de las sustancias aplicadas en el tejido corneal crean nuevos enlaces covalentes entre las fibrillas de colágeno, requiriendo la presencia de oxígeno en cantidades suficientes para participar en la reacción. Es posible aumentar la velocidad del proceso (alta fluencia) y disminuir la penetración del oxígeno debido al epitelio en el estroma, como sucede en el entrecruzamiento transepitelial ${ }^{19}$.

La concentración de oxígeno en la córnea se modula por la radiación y la temperatura de la radiación UVA, y se reduce rápidamente al comienzo de la exposición a UVA. Dado que la dependencia temporal de los mecanismos fotoquímicos, tanto del tipo I como del tipo II, con la riboflavina en la reticulación de la córnea a nivel estromal, así como su interacción en el epitelio, todavía debe entenderse mejor, pudiera considerarse que el tratamiento pulsado, al modificar las concentraciones de oxígeno, comparado con el convencional, pudiese haber influido en el defecto epitelial persistente del caso presentado.

\section{4)Conservadores de otros medicamentos y epitelio}

Además de los conservadores en los AINE, los presentes en los antibióticos y lubricantes, así como los esteroides tópicos, pueden contribuir a retrasar la velocidad de epitelización. Las quinolonas de cuarta generación, utilizadas con frecuencia por su amplio espectro, pueden influir debido a su toxicidad tanto corneal como conjuntival, ya sea por sus conservadores, como el cloruro de benzalconio ${ }^{20}$, reduciendo la velocidad de epitelización, o por la interacción directa del fármaco con el epitelio, que puede cristalizarse formando depósitos ${ }^{21}$. En nuestro caso se consideró esta posibilidad a la semana del defecto epitelial, y dado el amplio espectro que también puede cubrir la netilmicina ${ }^{22}$, junto con la menor toxicidad epitelial, se optó por esta opción, y además cambiar el lubricante a uno con un mecanismo de acción cicatricial libre de conservadores.

\section{5) Lente de contacto terapéutico}

Los lentes de contacto son uno de los aspectos más importantes en la cicatrización epitelial posoperatoria. Reducen el dolor generado por el roce del párpado mediante una barrera que impide el barrido mecánico de las capas superficiales epiteliales, así como el contacto directo con las terminaciones del plexo nervioso subepitelial.

$\mathrm{Si}$ el lente no es adaptado correctamente, es de un material con poca permeabilidad al oxígeno (hidrogel sin silicón) o se deposita en su interior un exceso de proteínas y lípidos, dará como resultado una marcada hipoxia con retraso en la migración celular epitelial. La absorción de proteínas se ve afectada por el contenido de agua del material y la interacción de la carga de la superficie con la carga de las proteínas de la película lagrimal. Los materiales para lentes con carga negativa muestran mayor afinidad por las proteínas con carga positiva, como la lisozima, la lactoferrina y la albúmina, por lo que la deposición total de proteínas en los lentes de hidrogel con alto contenido de agua, iónicos, es mayor (> $1500 \mu \mathrm{g}$ por lente) que en otros hidrogeles con bajo contenido de agua, no iónicos (10-20 $\mu \mathrm{g}$ por lente $)^{23}$. Aunque el lente debería permanecer hasta el cierre epitelial, en los casos en que se documentan datos de hipoxia desde las primeras 24 horas se ha recomendado una revisión más frecuente, incluso diaria, y con recambio del lente si es necesario. En nuestro caso, el paciente permaneció con un 
lente de contacto de hidrogel silicón con un $36 \%$ de contenido en agua, en el cual, aunque los depósitos no son tan frecuentes, tal vez no se documentaron de manera correcta y oportuna hasta los 7 días del posoperatorio, por lo que pudiera ser una de las causas importantes de la mala cicatrización.

\section{6)Anestésicos tópicos}

En algunos casos, con el uso excesivo de anestésicos tópicos, sobre todo cuando se llegan a proporcionar con uso libre en el posoperatorio (lo cual ya es poco frecuente, pero desafortunadamente es una práctica que aún persiste), se conoce un amplio espectro clínico de reacciones tóxicas, que incluyen queratopatía punteada, defecto epitelial persistente, infiltrados estromales, edema corneal, plegamiento de la membrana de Descemet, pérdida de células endoteliales, úlceras neurotróficas, lisis estromal, queratitis infecciosa y hasta perforaciones. No se utilizaron en el posoperatorio del caso clínico, pero se indagó sobre el número de ocasiones y la dosis durante el tratamiento transoperatorio, y consistieron únicamente en tetracaína tópica, $5 \mathrm{mg} / \mathrm{ml}$, una gota en cada ojo, previo al tratamiento.

\section{7)Edad}

La aplicación del entrecruzamiento en edades pediátricas es una práctica que ha aumentado de manera considerable en los últimos años ${ }^{24,25}$. Si comparamos las complicaciones significativas de los entrecruzamientos con los problemas resultantes de la progresión del queratocono y los riesgos de la queratoplastia en niños, además de los costos, la comparación favorece al entrecruzamiento ${ }^{26}$. Sin embargo, aunque la mayoría de los estudios están a favor por los resultados en el primer año, en los pacientes pediátricos no hay un efecto tan llamativo en el aplanamiento de la córnea como en los adultos, y faltan muchos datos a largo plazo. Los probables beneficios del tratamiento transepitelial en estas edades incluyen un riesgo posiblemente menor de queratitis infecciosa y una reducción del dolor, lo cual es en particular importante en los niños pequeños ${ }^{27}$. Al ser un tratamiento relativamente nuevo en pediatría, aún se esperan resultados a largo plazo sobre los efectos y las complicaciones relacionadas con la cicatrización tras los diferentes tipos de tratamientos transepiteliales y los distintos niveles de irradiación.

\section{Conclusiones}

Aunque la técnica de entrecruzamiento corneal transepitelial se ha considerado en general como segura y eficaz, es relevante tomar en cuenta todos los factores de riesgo previos al tratamiento, sobre todo en edades pediátricas, en las que la experiencia a largo plazo es menor que en los adultos. La revisión y el seguimiento del grado de epitelización durante los primeros días, el estado del lente de contacto, los conservadores en los medicamentos y las enfermedades en la superficie ocular pueden reducir el riesgo de fibrosis, infecciones y lisis corneal.

\section{Conflicto de intereses}

Los autores declaran no tener ningún conflicto de intereses.

\section{Responsabilidades éticas}

Protección de personas y animales. Los autores declaran que para esta investigación no se han realizado experimentos en seres humanos ni en animales.

Confidencialidad de los datos. Los autores declaran que han seguido los protocolos de su centro de trabajo sobre la publicación de datos de pacientes.

Derecho a la privacidad y consentimiento informado. Los autores han obtenido el consentimiento informado de los pacientes y/o sujetos referidos en el artículo. Este documento obra en poder del autor de correspondencia.

\section{Bibliografía}

1. Spoerl E, Schreiber J, Hellmund K, Seiler T, Knuschke P. Untersuchungenzur Verfestigung der Hornhaut am kaninchen. Ophthalmologe. 2000;97:203-6.

2. Wollensak G, Spoerl E, Seiler T. Riboflavin/ultraviolet-A-induced collagen cross-linking for the treatment of kertatoconus. Am J Ophthalmol. 2003;135:620-7

3. Meiri Z, Keren S, Rosenblatt A, Sarig T, Shenhav L, Varssano D. Efficacy of corneal collagen cross-linking for the treatment of keratoconus: a systematic review and meta-analysis. Cornea. 2016;35:417-28.

4. Chan C, Sharma M, Wachler B. Effect of inferior segment Intacs with and without C3-R on keratoconus. J Cataract Refract Surg. 2007; $33: 75-80$

5. lovineo A, Legare MI, Rootman DB, Yeung SN, Kim P, Rootman DS. Intracorneal ring segments implantation followed by same day photo-refractive keratectomy and corneal collagen cross-linking in keratoconus. J Refract Surg. 2011;27:915-8.

6. Gordillo $\mathrm{CH}$, Grandin JC, Zaldivar R, Zaldivar R, Lotfi AC. Efficacy of intrastromal corneal ring segments combined with flash collagen cross-linking in keratoconus. Cornea. 2017;36:144-7.

7. Kymionis GD, Grentzelos MA, Portaliou DM, Kankariya VP, Randleman JB. Corneal collagen cross-linking $(\mathrm{CXL})$ combined with refractive procedures for the treatment of corneal ectatic disorders: CXL plus. J Refract Surg. 2014;30:566-76.

8. Fadlallah A, Dirani A, Chelala E, Antonios R, Cherfan G, Jarade E. Non-topography-guided PRK combined with CXL for the correction of refractive errors in patients with early stage keratoconus. J Refract Surg. 2014;30:688-93.

9. Akbar B, Intisar-Ul-Haq R, Ishaq M, Fawad A, Arzoo S, Siddique K. Comparison of transepithelial corneal crosslinking with epithelium-off crosslinking (epithelium-off $\mathrm{CXL}$ ) in adult Pakistani population with progressive keratoconus. Taiwan J Ophthalmol. 2017;7:185-90. 
10. Medeiros CS, Giacomin NT, Bueno RL, Ghanem RC, Moraes HV Jr, Santhiago MR. Accelerated corneal collagen crosslinking: technique, efficacy, safety, and applications. J Cataract Refract Surg. 2016; 42:1826-35.

11. Leccisotti A, Islam T. Transepithelial corneal collagen cross-linking in keratoconus. J Refract Surg. 2010;26:942-8.

12. Li W, Wang B. Efficacy and safety of transepithelial corneal collagen crosslinking surgery versus standard corneal collagen crosslinking surgery for keratoconus: a meta-analysis of randomized controlled trials. BMC Ophthalmol. 2017;17:262.

13. Liu Y, Liu Y, Zhang YN, Li AP, Zhang J, Liang QF, et al. Systematic review and Meta-analysis comparing modified cross-linking and standard cross-linking for progressive keratoconus. Int J Ophthalmol. 2017 10:1419-29.

14. Shalchi Z, Wang X, Nanavaty MA. Safety and efficacy of epithelium removal and transepithelial corneal collagen crosslinking for keratoconus. Eye (Lond). 2015;29:15-29.

15. Mazzotta C, Hafezi F, Kymionis G, Caragiuli S, Jacob S, Traversi C, et al. In vivo confocal microscopy after corneal collagen crosslinking. Ocul Surf. 2015;13:298-314

16. Cerman E, Özcan DÖ, Toker E. Sterile corneal infiltrates after corneal collagen cross-linking: evaluation of risk factors. Acta Ophthalmol. 2017:95:199-204

17. Lin JC, Rapuano CJ, Laibson PR, Eagle RC, Cohen EJ. Corneal melting associated with use of topical nonsteroidal anti-inflammatory drugs after ocular surgery. Arch Ophthalmol. 2000;118:1129-32.
18. Taneri S, Oehler S, Lytle G, Dick HB. Evaluation of epithelial integrity with various transepithelial corneal cross-linking protocols for treatment of keratoconus. J Ophthalmol. 2014;2014:614380.

19. Richoz O, Hammer A, Tabibian D, Gatzioufas Z, Hafezi F. The biomechanical effect of corneal collagen cross-linking (CXL) with riboflavin and UV-A is oxygen dependent. TransI Vis Sci Technol. 2013;2:6.

20. Sosa $A B$, Epstein SP, Asbell PA. Evaluation of toxicity of commercial ophthalmic fluoroquinolone antibiotics as assessed on immortalized corneal and conjunctival epithelial cells. Cornea. 2008;27:930-4.

21. Elia M, Khodadadeh S, Chow J. Corneal crystalline deposits associated with topically applied gatifloxacin. Cornea. 2014:33:638-9.

22. Alcántara-Castro M, Flores V. Susceptibilidad a netilmicina en 400 cepas bacterianas aisladas de infecciones oculares. Rev Mex Oftalmol. 2009;83:1-5.

23. Omali NB, Subbaraman LN, Coles-Brennan C, Fadli Z, Jones LW. Biological and clinical implications of lysozyme deposition on soft contact lenses. Optom Vis Sci. 2015;92:750-7.

24. Vinciguerra P, Albé E, Frueh BE, Trazza S, Epstein D. Two-year corneal cross-linking results in patients younger than 18 years with documented progressive keratoconus. Am J Ophthalmol. 2012;154:520-6.

25. Caporossi A, Mazzotta C, Baiocchi S, Caporossi T, Denaro R, Balestrazzi A. Riboflavin-UVA-induced corneal collagen cross-linking in pediatric patients. Cornea. 2012;31:227-31.

26. Badawi AE. Accelerated corneal collagen cross-linking in pediatric keratoconus: one year study. Saudi J Ophthalmol. 2017;31:11-8.

27. McAnena L, Doyle F, O'Keefe M. Cross-linking in children with keratoconus: a systematic review and meta-analysis. Acta Ophthalmol. 2017;95:229-39. 